\title{
Use of Mobile Communication in Data-Intensive Wireless Networks
}

\author{
Nuthanapati Jyothsna ${ }^{1}$ Reddy Sagar A C ${ }^{2}$ Ravi Kumar G ${ }^{3}$ \\ ${ }^{I}$ PG Student, Siddartha Institute of Science and Technology, Puttur chittoor(d) A.P, \\ ${ }^{2}$ Assistant Professor, Siddartha Institute of Science and Technology, Puttur chittoor(d) A.P, \\ ${ }^{3}$ Assistant Professor, Siddartha Institute of Science and Technology, Puttur chittoor(d) A.P,
}

\begin{abstract}
Wireless Networks are increasingly used in different types of data-intensive applications scenarios such as micro-weather monitoring, meticulousness agriculture, and audio/video observation. The sensor nodes are minute and limited in power. Sensor types vary according to the application of WN. Whatever be the application the key challenge is to broadcast all the data generated within an application's life span to the base station in the face of the fact that sensor nodes have limited power supplies. The concept of mobile communication is that the mobile nodes change their locations so as to minimize the total energy consumed by both wireless transmission and locomotion. The predictable methods, however, do not take into account the energy level, and as a result they do not always extend the network lifetime.
\end{abstract}

Keywords: Data-intensive; Energy; communication; routing tree; WN

\section{INTRODUCTION}

Sensors have the capabilities of doing sensing, data processing, and wirelessly transmitting collected data back to base stations by way of multiple-hop relay. Sensor itself supplies necessary operation with limited battery energy.

Those operations that consume energy are transmitting and receiving data, running applications, measuring power, and even staying in standby mode. Among others, data transmission consumes the most energy. A wireless sensor network (WSN) consists of spatially distributed autonomous sensors to monitor physical or environmental conditions,

such as temperature, sound, vibration, pressure humidity, motion or pollutants and to cooperatively pass their data through the network to a main location[1,2]. The more modern networks are bi-directional, also enabling control of sensor activity. The development of wireless sensor networks was

motivated by military applications such as battlefield surveillance; today such networks are used in many industrial and consumer applications, such as industrial process monitoring and control, machine health monitoring, and so on. Figure 1 shows an example of a wireless sensor network.

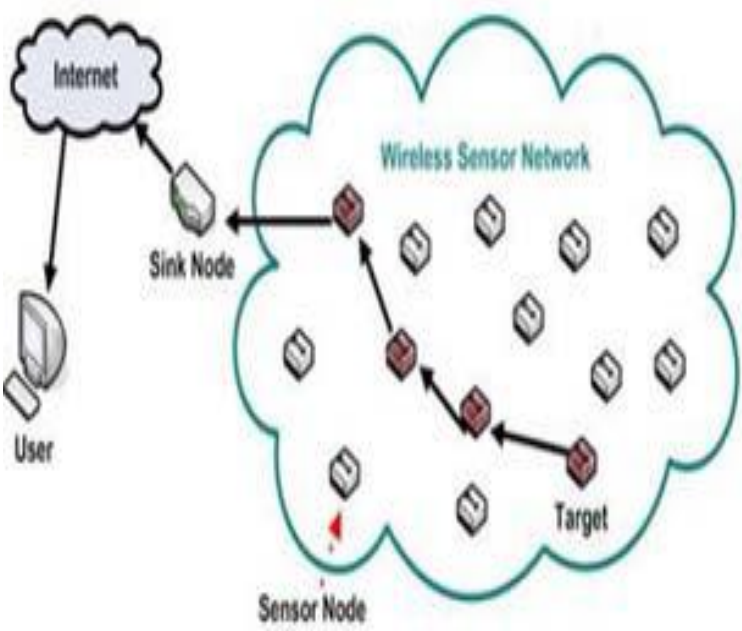

Figure 1: An example of Wireless Sensor Network

Recent advancement in mobile sensor platform technology has been taken into attention that mobile elements are utilized to improve the WSN's performances such as coverage, connectivity, reliability and energy efficiency. The concept of mobile relay is that the mobile nodes change their locations so as to minimize the 
total energy consumed by both wireless transmission and locomotion. The conventional methods, however, do not take into account the energy level, and as a result they do not always prolong the network lifetime.

\section{RELATED WORK}

Analyzing the three different approaches: Mobile base stations, data mules and mobile relays. All the three approaches use mobility to reduce energy consumption in wireless networks.

\section{A. Mobile Base Station}

A mobile base station is a sensor node collects the data by moving around the network from the nodes [4]. In some work, in order to balance the transmission load, all nodes are performing multiple hop transmissions to the base station. The goal is to rotate the nodes which are close to the base station. Before the nodes suffer buffer overflows, the base station computes the mobility path to collect data from the visited nodes. Several rendezvous based data collection algorithms are proposed, where the mobile base station only visits a selected set of nodes referred to as rendezvous points within a deadline and the rendezvous points buffer the data from sources. High data traffic towards the base station is always a threat to the networks life time [5]. The battery life of the base station gets depleted very quickly due to the sensor nodes which are located near to the base station relay data for large part of the network. The proposed solution includes the mobility of the base station such that nodes located near base station changes over time. All the above approaches incur high latency due to the low to moderate speed of mobile base stations. Figure 2 shows Mobile base station

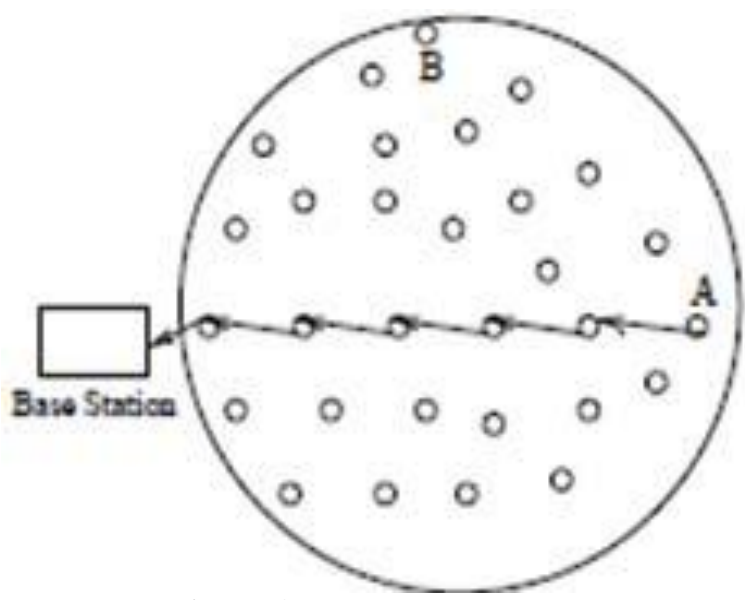

Figure 2: Mobile base station

\section{B. Data Mules}

Data mules are another form of base stations. They gather data from the sensors and carry it to the sink. The data mule collects the data by visiting all the sources and then transmits it to the static base station through the network. In order to minimize the communication and mobility energy

Consumption the mobility paths are determined. In paper [6] the author analyses an architecture based on mobility to address the energy efficient data collection problem in a sensor network. This approach utilizes the mobile nodes as forwarding agents. As a mobile node moves in close propinquity to sensors, data is transmitted to the mobile node for later dumps at the destination. In the MULE architecture sensors transmit data only over a short range that requires less transmission power. However, latency is increased because a sensor has to wait for a mule before its data can be Delivered. Figure 3. The three tiers of the MULE architecture. The Mule architecture has high latency and this limits its applicability to real time applications (although this can be mitigated by collapsing the MULE and access point tiers). The system requires sufficient mobility. For example, mules may not arrive at a sensor or after picking the data may not reach near an accesspoint to deliver it. Also, data may be lost because of radio-communication errors or mules crashing. To improve data delivery, higher-level protocols need to be incorporated in the MULE architecture. Data

mules also introduce large delays like base stations since sensors have to wait for a mule to pass by before initiating their transmission. 


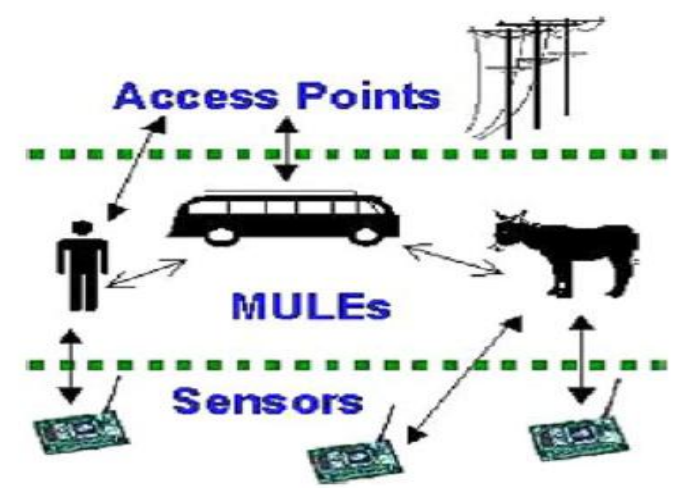

Figure 3: The three tiers of the MULE architecture

\section{Mobile Relay}

In this approach, the network consists of three nodes such as mobile relay nodes along with static base station and data sources. To reduce the transmission cost relay nodes do not transport data rather it will move to different locations. We use the mobile relay approach in this work. In [7] author showed that an iterative mobility algorithm where each relay node moves to the midpoint of its neighbors converges on the optimal solution for a single routing path This paper presents mobility control scheme for improving communication performance in WSN. The objectives of the paper are

1) Analyze when controlled mobility can improve fundamental networking performance metrics such as power efficiency and robustness of communications

2) Provide initial design for such networks. Mobile nodes move to midpoint of the neighbors only when movement is beneficial.

Unlike mobile base stations and data mules, our approach reduces the energy consumption of both mobility and transmission. Our approach also relocates each mobile relay only once immediately after deployment. The paper study the energy optimization problem that accounts for energy costs associated with both communication and physical node movement. Unlike previous mobile relay schemes the proposed solution consider all possible locations as possible target locations for a mobile node instead of just the midpoint of its neighbors.

\section{PROPOSED WORK}

We use low-cost disposable mobile relays to reduce the total energy consumption of data-intensive WSNs. Different from mobile base station or data mules, mobile relays do not transport data; instead, they move to different locations and then remain stationary to forward data along the paths from the sources to the base station. Thus, the communication delays can be significantly reduced compared with using mobile sinks or data mules. Moreover, each mobile node performs a single relocation unlike other approaches which require repeated relocations. Figure 4 shows Proposed Network.

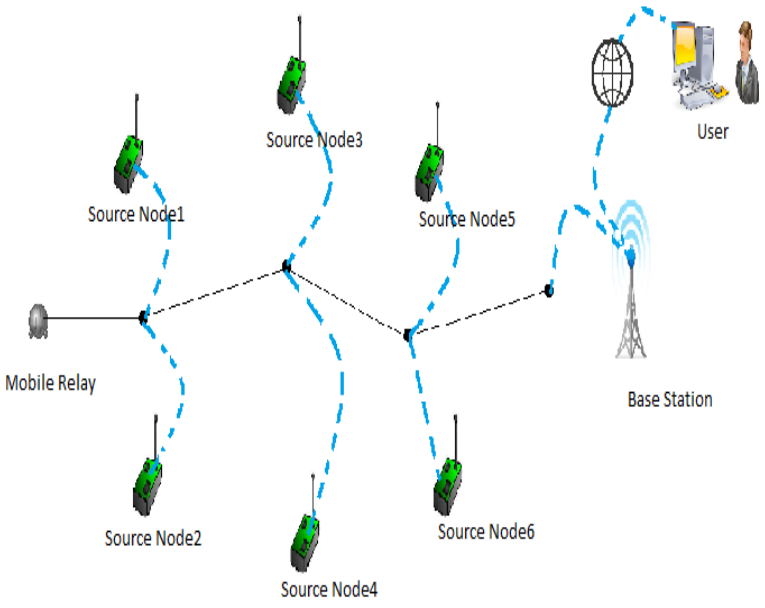

Figure4: Proposed Network 
The network consists of mobile relay nodes along with static base station and data sources. Relay nodes do not transport data; instead, they move to different locations to decrease the transmission costs. We use the mobile relay approach in this work. Goldenberg et al. [13] showed that an iterative mobility algorithm where each relay node moves to the midpoint of its neighbors converges on the optimal solution for a single routing path. However, they do not account for the cost of moving the relay nodes. In mobile nodes decide to move only when moving is beneficial, but the only position considered is the midpoint of neighbors.

The sink is the point of contact for users of the sensor network. Each time the sink receives a question from a user, it first translates the question into multiple queries and then disseminates the queries to the corresponding mobile relay, which process the queries based on their data and return the query results to the sink. The sink unifies the query results from multiple storage nodes into the final answer and sends it back to the user.

The source nodes in our problem formulation serve as storage points which cache the data gathered by other nodes and periodically transmit to the sink, in response to user queries. Such a network architecture is consistent with the design of storage centric sensor networks. Our problem formulation also considers the initial positions of nodes and the amount of data that needs to be transmitted from each storage node to the sink.

We consider the sub problem of finding the optimal positions of relay nodes for a routing tree given that the topology is fixed. We assume the topology is a directed tree in which the leaves are sources and the root is the sink. We also assume

that separate messages cannot be compressed or merged; that is, if two distinct messages of lengths $\mathrm{m} 1$ and $\mathrm{m} 2$ use the same link $\left(\mathrm{s}_{\mathrm{i}}, \mathrm{s}_{\mathrm{j}}\right)$ on the path from a source to a sink, the total number of bits that must traverse link $\left(\mathrm{s}_{\mathrm{i}}, \mathrm{s}_{\mathrm{j}}\right.$ ) is $m_{1}+m_{2}$.

\section{a) Energy Optimization Framework}

In this section, we formulate the problem of Optimal Mobile Relay Configuration (OMRC) in dataintensive WSNs. Unlike mobile base stations and data mules, our OMRC problem considers the energy consumption of both mobility and transmission. The Optimal Mobile Relay Configuration (OMRC) problem is challenging because of the dependence of the solution on multiple factors such as the routing tree topology and the amount of data transferred through each

link. For example, when transferring little data, the optimal configuration is to use only some relay nodes at their original positions.

Assume the network consists of one source $s_{i}-1$, one mobile relay node $s_{i}$ and one sink $s_{i}+1$. Let the original position of a node sj be $\mathrm{o}_{\mathrm{j}}=\left(\mathrm{p}_{\mathrm{j}}, \mathrm{q}_{\mathrm{j}}\right)$, and let $\mathrm{u}_{\mathrm{j}}=\left(\mathrm{x}_{\mathrm{j}}, \mathrm{y}_{\mathrm{j}}\right)$ its final position in configuration $\mathrm{U}$. According to our energy models, the total transmission and movement energy cost incurred by the mobile relay node $\mathrm{s}_{\mathrm{i}}$ is $\mathrm{c}_{\mathrm{i}}(\mathrm{U})=\mathrm{k}\left\|\mathrm{u}_{\mathrm{i}}-\mathrm{o}_{\mathrm{i}}\right\|+\mathrm{am}+\mathrm{b}\left\|\mathrm{u}_{\mathrm{i}}+1-\mathrm{u}_{\mathrm{i}}\right\|^{2} \mathrm{~m}$

Now We need to compute a position ui for si that minimizes $C_{i}(U)$ assuming that $\mathrm{u}_{\mathrm{i}}-1=\mathrm{o}_{\mathrm{i}}-1$ and $\mathrm{u}_{\mathrm{i}}+1=\mathrm{o}_{\mathrm{i}}+1$; that is, node $\mathrm{s}_{\mathrm{i}}$ 's neighbors remain at the same positions in the final configuration $\mathrm{U}$. We calculate position $u_{i}=$ $\left(\mathrm{x}_{\mathrm{i}}, \mathrm{y}_{\mathrm{i}}\right)$ for node $\mathrm{s}_{\mathrm{i}}$ by finding the values for $\mathrm{xi}$ and $\mathrm{y}_{\mathrm{i}}$ where the partial

derivatives of the cost function $C_{i}(U)$ with respect to $x_{i}$ and $y_{i}$ become zero. Position $U_{i}$ will be toward the midpoint of positions $u_{i}-1$ and $u_{i}+1$. The partial derivatives at $x_{i}$ and $y_{i}$, respectively are defined as follows:

$\delta \mathrm{C}_{\mathrm{i}}(\mathrm{U})$

$\delta \mathrm{x}_{\mathrm{i}}$

$\left(\mathrm{x}_{\mathrm{i}}-\mathrm{p}_{\mathrm{i}}\right)$

$\left.+x_{i}-p_{i}\right)^{2}+\left(y_{i}-q_{i}\right) 2$

$\delta \mathrm{C}_{\mathrm{i}}(\mathrm{U})$

$\delta$ yi

(yi - qi)

$+\mathrm{k}--$

$\sqrt{ }\left(x_{i}-p i\right) 2+(y i-q i) 2$

and rms do not have to be defined. Do not use abbreviations in the title or heads unless they are unavoidable. 


\section{b) Tree Optimization Algorithm}

In this section, we consider the sub problem of finding the optimal positions of relay nodes for a routing tree given that the topology is fixed. We assume the topology is a directed tree in which the leaves are sources and the root is the sink. We also assume that separate messages cannot be compressed or merged; that is, if two distinct messages of lengths $\mathrm{m} 1$ and $\mathrm{m} 2$ use the same link $\left(\mathrm{s}_{\mathrm{i}}, \mathrm{s}_{\mathrm{j}}\right)$ on the path from a source to a sink, the total number of bits that must traverse $\operatorname{link}\left(\mathrm{s}_{\mathrm{i}}, \mathrm{s}_{\mathrm{j}}\right)$ is $\mathrm{m}_{1}+\mathrm{m}_{2}$. Let the network consists of multiple sources, one relay node and one sink such that data is transmitted from each source to the relay node and then to the sink. We modify our solution as follows. Let $\mathrm{s}_{\mathrm{i}}$ be the mobile relay node, $\mathrm{S}\left(\mathrm{s}_{\mathrm{i}}\right)$ the set of source nodes transmitting to $\mathrm{s}_{\mathrm{i}}$ and $\mathrm{sd}_{\mathrm{i}}$ the sink collecting nodes from $\mathrm{s}_{\mathrm{i}}$. The cost incurred by $\mathrm{s}_{\mathrm{i}}$ in this configuration $\mathrm{U}$ is:

$c_{i}(U)=k\left\|u_{i}-o_{i}\right\|+a_{i}+b m_{i}\left\|u_{i}+1-u_{i}\right\|^{2}$

Algorithm 1

procedure OPTIMALPOSITIONS $\left(\mathrm{U}_{0}\right)$

converged $\leftarrow$ false;

$\mathrm{j} \leftarrow 0$;

repeat

anymove $\leftarrow$ false;

$\mathrm{j} \leftarrow \mathrm{j}+1$

$\triangleright$ Start an even iteration followed by an odd iteration

for idx $=2$ to 3 do

for $\mathrm{i}=\mathrm{idx}$ to $\mathrm{n}$ by 2 do

$\left(\mathrm{u}_{\mathrm{i}}^{\mathrm{j}}\right.$, moved $) \leftarrow \operatorname{LOCALPOS}\left(\mathrm{o}_{\mathrm{i}}, \mathrm{S}\left(\mathrm{s}_{\mathrm{i}}\right), \mathrm{sd}_{\mathrm{i}}\right)$;

anymove $\leftarrow$ anymove OR moved

end for

end for

converged $\leftarrow$ NOT anymove

until converged

end procedure

Our algorithm starts by an odd/even labeling step followed by a weighting step. To obtain consistent labels for nodes, we start the labeling process from the root using a breadth first traversal of the tree. The root gets labeled as even. Each of its children gets labeled as odd. Each subsequent child is then given the opposite label of its parent. We define mi, the weight of a node $\mathrm{s}_{\mathrm{i}}$, to be the sum of message lengths over all paths passing through $s_{\mathrm{i}}$. This computation starts from the sources or leaves of our routing tree. Initially, we know mi $=$ Mi for each source leaf node $s_{i}$. For each intermediate node $s_{i}$, we compute its weight as the sum of the weights of its children. Once each node gets a weight and a label, we start our iterative scheme. In odd iterations $j$, the algorithm

computes a position $\mathrm{u}^{\mathrm{j}}{ }_{\mathrm{i}}$ for each odd-labeled node $\mathrm{s}_{\mathrm{i}}$ that minimizes $\mathrm{C}_{\mathrm{i}}\left(\mathrm{U}_{\mathrm{j}}\right)$ assuming that

$\mathrm{u}^{\mathrm{j}}{ }_{\mathrm{i}}-1=\mathrm{u}^{\mathrm{j}-1}{ }_{\mathrm{i}}-1$ and $\mathrm{u}^{\mathrm{j}}{ }_{\mathrm{i}+1}=\mathrm{u}^{\mathrm{j}-1}{ }_{\mathrm{i}+1}$ that is, node $\mathrm{s}_{\mathrm{i}}{ }^{\prime}$ s even numbered neighboring nodes remain in place in configuration $U_{j}$. In even-numbered iterations, the controller does the same for even-labeled nodes. The algorithm behaves this way because the optimization of $\mathrm{u}^{j}{ }_{\mathrm{i}}$ requires a fixed location for the child nodes and the parent of $\mathrm{s}_{\mathrm{i}}$. By alternating between optimizing for odd and even labeled nodes, the algorithm guarantees that the node $\mathrm{s}_{\mathrm{i}}$ is always making progress towards the optimal position ui. Our iterative algorithm is shown in algorithm1.

\section{CONCLUSION}

The main objective of this paper is energy conservation which is holistic in that the total energy consumed by both mobility of relays and wireless transmissions is minimized, which is in contrast to existing mobility approaches that only

minimize the transmission energy consumption. The tradeoff in energy consumption between mobility and transmission is exploited by configuring the positions of mobile relays. We

develop two algorithms that iteratively refine the

configuration of mobile relays. The first improves the tree topology by adding new nodes. It is not guaranteed to find the optimal topology. The second improves the routing tree by relocating nodes without changing the tree topology. It converges to the optimal node positions for the given topology. Our algorithms have efficient distributed implementations that require only limited, localized synchronization. 


\section{REFERENCES}

[1] I.F. Akyildiz, W. Su, Y. Sankarasubramaniam, and E.Cayirci, "A Survey on Sensor Networks," IEEE Comm.Magazine, vol. 40, no. 8, pp. 102-114, Aug. 2002.

[2] Shio Kumar Singh, M.P. Singh, and D.K. Singh, “Applications, Classifications, and Selections of Routing Protocols for Wireless Sensor Networks" International Journal of Advanced Engineering Sciences and Technologies (IJAEST), November 2010, vol. 1, issue no. 2, pp. 85-95.

[3] Ganesan, B. Greenstein, D. Perelyubskiy, D. Estrin, and J.Heidemann, "An Evaluation of MultiResolution Storage for Sensor Networks," Proc. First Int'l Conf. Embedded Networked Sensor Systems (SenSys), 2003.

[4] Fatme El-Moukaddem, Eric Torng, Guoliang Xing" Mobile Relay Configuration in Data-intensive Wireless Sensor Networks" in IEEE Transactions on Mobile computing, 2013.

[5] J. Luo and J.-P. Hubaux, Joint Mobility and Routing for Lifetime Elongation in Wireless Sensor Networks, in INFOCOM, 2005.

[6] S. Jain, R. Shah, W. Brunette, G. Borriello, and S. Roy,Exploiting Mobility for energy Cient Data Collection in Wireless Sensor Networks, MONET,vol. 11, pp. 327339, 2006.

[7] K. Goldenberg, J. Lin, and A. S. Morse, Towards Mobility as a Network Control Primitive, in MobiHoc, 2004, pp. 163174.

[8] Tang and P. K. McKinley, Energy Optimization Under Informed Mobility, IEEE Trans. Parallel Distrib. Syst., vol. 17,pp. 947962, 2006.

[9] Fatme El-Moukaddem, Eric Torng, and Guoliang Xing, Member, IEEE "Mobile Relay Configuration in Data- Intensive Wireless Sensor Networks". 